\section{Enteroparasitoses e onicomicoses em manipuladores de alimentos do município de Ribeirão Preto, SP, Brasil}

\section{Enteroparasitosis and onychomycosis in food handlers in the city of Ribeirão Preto, SP, Brasil}

\author{
Jaqueline Otero Silva' \\ Divani M. Capuano' \\ Osvaldo M. Takayanagui² \\ Eugênio Giacometti Júnior ${ }^{3}$ \\ ${ }^{1}$ Seção de Biologia Médica do Instituto Adolfo Lutz - Laboratório I de Ribeirão \\ Preto \\ ${ }^{2}$ Departamento de Neurologia da Faculdade de Medicina de Ribeirão Preto - \\ USP \\ ${ }^{3}$ Unidade Básica de Saúde Castelo Branco da Secretaria Municipal de Saúde de \\ Ribeirão Preto
}

Trabalho realizado no Instituto Adolfo Lutz - Laboratório I de Ribeirão Preto.

\section{Resumo}

A maioria das doenças veiculadas por alimentos de origem microbiana deve-se à manipulação inadequada dos mesmos. A identificação de manipuladores de alimentos portadores de agentes patogênicos que podem ser propagados para os alimentos é uma ferramenta útil na prevenção da contaminação dos mesmos. Objetivos: Identificar a presença de enteroparasitoses e onicomicoses em manipuladores de alimentos de Ribeirão Preto, contribuindo assim com as ações de vigilância sanitária. Métodos: Foram analisadas entre julho e novembro de 2000, 69 amostras de fezes e 23 de unhas de 23 manipuladores de alimentos de Ribeirão Preto. As fezes foram submetidas aos métodos de KATO e da sedimentação espontânea. Na análise micológica, cada amostra de raspado de unha foi submetida à clarificação com KOH $30 \%$ por 30 minutos para estudo microscópico (exame direto) e cultura em ágar Sabouraud acrescido de $100 \mathrm{mg} / \mathrm{L}$ de cloranfenicol e posterior incubação a $28^{0}$ $30^{\circ} \mathrm{C}$ por até 30 dias, verificando-se o crescimento semanalmente. Resultados: Dos trabalhadores avaliados, $04(17,4 \%)$ apresentaram parasitismo intestinal, $08(34,8 \%)$ micoses e em $01(4,3 \%)$ foi constatada infecção concomitante por enteroparasitas e micose. Conclusões: Para evitar as doenças transmitidas por alimentos é necessária a implementação conjunta de várias ações, incluindo a identificação dos portadores de agentes patogênicos que possam ser propagados para os alimentos através da manipulação.

Palavras-chave: Alimentos. Prevenção. Manipuladores de alimentos. Enteroparasitoses. Micoses. 


\section{Abstract}

Most microbial food-borne illnesses are due to inappropriate food handling. The identification of food handlers contaminated with pathogenic organisms that can be transferred to food is a useful tool in the prevention of food contamination. Objective: This study aimed to identify the presence of enteroparasitosis and onychomycosis in food handlers in Ribeirão Preto, in order to contribute to sanitary surveillance actions. Methods: From July to November 2000, 69 fecal and 23 nail samples of 23 food handlers in Ribeirão Preto were examined. Feces were submitted to the KATO's and spontaneous sedimentation method. In the mycological analysis, every nail scraping sample was submitted to clarification with $30 \% \mathrm{KOH}$ for 30 minutes for the microscopic study (direct examination) and culture in Sabouraud agar with $100 \mathrm{mg} / \mathrm{L}$ of chloramphenicol, and later incubation at 28$30^{\circ} \mathrm{C}$. Yeast growth was checked weekly. Results: Among the workers evaluated, 04 (17.4\%) presented intestinal parasitism, 08 (34.8\%) mycosis, and 01 (4.3\%), concurrent enteroparasitosis infection and mycosis. Conclusions: To prevent food-borne illnesses, the joint implementation of some actions is necessary, including the identification of pathogenic agents that can be transmitted by food, through handling.

Keywords: Food. Prevention. Food handlers. Enteroparasitosis. Mycoses.

\section{Introdução}

A oferta de alimentos isentos de agentes patogênicos que possam pôr em risco a saúde do consumidor assumiram mundialmente uma grande relevância em saúde pública. Nas últimas décadas, tem-se observado um aumento das doenças transmitidas por alimentos, relacionado a vários fatores, tais como o desenvolvimento econômico, a globalização do comércio de alimentos, a intensificação da urbanização e a modificações dos hábitos alimentares dos consumidores, com aumento do consumo de alimentos frescos ou "in natura", preferência por alimentos prontos ou semi-prontos e o consumo de refeições fora do domicílio ${ }^{1,2}$. O Centers for Disease Control and Prevention (CDC $)^{1}$ estima que anualmente, nos Estados Unidos da América, as doenças transmitidas por alimentos acometem 76 milhões de pessoas, sendo que mais de 300 mil são hospitalizadas e 500 vão a óbito. Os grupos populacionais mais atingidos são as crianças, os idosos e os imunocomprometidos $^{1,3}$. No Brasil, dentre as doenças transmitidas por alimentos, entre $1999 \mathrm{e}$ 2002, foram notificados 176 surtos por Salmonella sp, 60 por Staphylococcus aureus, 09 por coliformes fecais e 06 por Shigella sp ${ }^{4}$. Entre 1999 e 2002 no Estado de São Paulo, foram notificados ao Centro de Vigilância Epidemiológica (CVE), 878 surtos de doenças transmitidas por alimentos, com 20.471 casos. Os agentes etiológicos incluíram bactérias $(24,6 \%)$, vírus $(26,8 \%)$ e parasitas $(2,5 \%)^{5}$. Entre as principais causas de doenças de origem microbiana veiculadas por alimentos está a manipulação inadequada dos mesmos ${ }^{2}$. Portanto, as pessoas que manipulam alimentos desempenham uma função importante na preservação da higiene dos mesmos, pois podem representar uma importante fonte de transmissão de vários patógenos. Entre estes, os parasitas intestinais encontram-se distribuídos praticamente por todo o mundo, registrando elevados coeficientes de prevalência em po- 
pulações com precárias condições higiênico-sanitárias. Estudos conduzidos em comunidades carentes da África, Turquia, Vietnã, México e Brasil, demonstraram taxas de infestação parasitária de 55,8\%, $37,2 \%, 88,0 \%, 67,0 \%$ e $79,0 \%$, respectivamente, com maiores freqüências de Ascaris lumbricoides, ancilostomídeos, Trichuris trichiura e Giardia lamblia ${ }^{6-10}$. Contudo, as infecções parasitárias intestinais são em sua maioria assintomáticas e, quando determinam alguma sintomatologia, esta é geralmente discreta e inespecífica, não sendo muitas vezes diagnosticada. Portanto, o manipulador de alimentos parasitado e assintomático pode representar uma fonte de transmissão duradoura, podendo propagar os enteroparasitas para os alimentos através das mãos contaminadas. Assim, a identificação da ocorrência de enteroparasitoses em manipuladores pode contribuir na prevenção da contaminação de alimentos.

As micoses em geral, apesar de não serem consideradas doenças de notificação obrigatória em nosso meio, são reconhecidas como problema de saúde pública em muitas partes do mundo. Sua prevalência está diretamente ligada às condições socioeconômicas, baixa higiene, condições sanitárias, assim como idade, sexo, ocupação e profissão ${ }^{11}$. Com isso, embora a onicomicose não seja uma infecção relacionada com transmissão alimentar, o seu controle nos manipuladores de alimentos, torna-se importante para a manutenção de uma higiene adequada. A presença de micose ungueal pode também servir como porta de entrada para outros microrganismos, favorecendo a instalação de uma infecção secundária por bactérias. Estas poderão ser propagadas para o alimento, contribuindo com a sua contaminação ${ }^{12}$. Um dos fatores que influenciam na aderência e na multiplicação das bactérias na pele é a esfoliação da mesma. Portanto, a existência de onicomicoses pode favorecer o crescimento bacteriano no local das lesões, particularmente do Staphylococcus aureus, organismo originário essencialmente da pele do homem e dos animais.

Neste contexto, para garantir a inocuidade dos alimentos, é de suma importância em saúde pública identificar manipuladores portadores de agentes que possam ser propagados para os alimentos, contribuindo para evitar a contaminação dos mesmos. Os objetivos deste estudo foram identificar a presença de enteroparasitoses e onicomicoses em manipuladores de alimentos de Ribeirão Preto, desse modo colaborando com as ações de vigilância sanitária, visando garantir a qualidade dos alimentos.

\section{Metodologia}

\section{População estudada}

Para efeito de inspeção sanitária de alimentos, é considerado manipulador qualquer pessoa que entre em contato direta ou indiretamente com os mesmos. Segundo a Portaria CVS-6/9913, "o controle de saúde clínico exigido pela Vigilância Sanitária objetiva a saúde do trabalhador e a sua condição para estar apto para o trabalho, não podendo ser portador aparente ou inaparente de doenças infecciosas ou parasitárias. Para isso devem ser realizados os exames médicos admissionais, periódicos, dando ênfase aos parâmetros preconizados neste regulamento, acompanhados das análises laboratoriais como: hemograma, coprocultura, coproparasitológico e VDRL, devendo ser realizadas outras análises de acordo com avaliação médica. Não devem manipular alimentos os funcionários que apresentarem feridas, lesões, chagas ou cortes nas mãos e braços, ou gastroenterites agudas ou crônicas (diarréia ou disenteria), assim como os que estiverem acometidos de infecções pulmonares ou faringites."

Participaram deste estudo retrospectivo 23 indivíduos que, entre julho e novembro de 2000, procuraram o Ambulatório de Saúde do Trabalhador da Unidade Básica Distrital de Saúde (UBDS) do Bairro Castelo Branco, em Ribeirão Preto, para ob- 
tenção da Carteira de Saúde. Esta unidade de saúde atende os habitantes da região sudeste do município, delimitada segundo critérios da Secretaria Municipal de Saúde de Ribeirão Preto. O critério de seleção dos indivíduos foi a necessidade da coleta simultânea de raspado de unha e parasitológico de fezes. Dados como idade, sexo, local de trabalho, foram obtidos através do protocolo de solicitação de exames preenchido pelo médico do trabalho no momento da consulta.

\section{Coleta das amostras}

Na pós consulta, uma auxiliar de enfermagem da UBDS devidamente treinada colheu os raspados de unha e forneceu orientações quanto à coleta e conservação das fezes. Em dias alternados, em suas respectivas residências, os manipuladores colheram três amostras de fezes em frascos limpos, sem adição de conservantes, mantendo-os em geladeira até a entrega na UBDS. As amostras biológicas coletadas e os protocolos de exames foram enviados ao Instituto Adolfo Lutz . Foram obtidas 23 amostras de raspados de unha e 69 de fezes.

\section{Análise Laboratorial}

$\mathrm{Na}$ análise coproparasitológica foram utilizados os métodos de $\mathrm{KATO}^{14}$ (2 lâminas por amostras de fezes) e o da sedimentação espontânea. As lâminas de KATO foram examinadas sob microscopia óptica logo após o seu preparo. Após no mínimo 16 horas de repouso, o sedimento obtido foi transferido para uma lâmina, sendo posteriormente adicionado lugol e examinado ao microscópio óptico sob ocular de 10X e 40X. $\mathrm{Na}$ análise micológica, cada amostra de raspado de unha foi submetida à clarificação com $\mathrm{KOH} 30 \%$ por 30 minutos para estudo microscópico (exame direto). A cultura foi realizada em duplicata em ágar Sabouraud acrescido de $100 \mathrm{mg} / \mathrm{L}$ de cloranfenicol os quais foram incubados a $28^{0}-30^{\circ} \mathrm{C}$ por até 30 dias, verificando-se o crescimento semanalmente. A identificação do agente etiológico foi baseado no estudo de suas características morfológicas em ágar fubá, produção de tubo germinativo em soro humano ou animal e provas bioquímicas utilizando método auxonográfico e zimográfico. A interpretação dos resultados para identificação da levedura foi baseada em Kurtzman \& Fell ${ }^{15}$.

Os resultados dos exames coproparasitológico e micológico foram enviados ao médico do trabalho para prescrição de terapia adequada, quando necessária. A emissão final da carteira de saúde de portadores de enteroparasitas foi condicionada à normalização de novo exame coproparasitológico após tratamento.

\section{Resultados}

Entre os trabalhadores investigados, 16 pertenciam ao sexo feminino e 07 ao masculino, com idade entre 18 a 60 anos (média de 39,3 anos).

O total de $04(17,4 \%)$ indivíduos apresentou parasitismo intestinal, sendo um deles poliparasitado. Consideramos como resultados positivos os protozoários comensais Entamoeba coli e Endolimax nana, pois os indivíduos parasitados foram temporariamente afastados do trabalho e receberam tratamento terapêutico pelo médico do trabalho. A presença de micoses foi constatada em 08 (34,8\%) indivíduos, com um caso de infecção concomitante apresentado por um funcionário de restaurante com 20 anos de idade. Os resultados encontrados segundo a idade, sexo e o local de trabalho dos manipuladores estão apresentados na Tabela 1.

\section{Discussão}

Diferentes métodos coprológicos têm sido adotados em estudos sobre a prevalência das enteroparasitoses em diversos grupos populacionais. Nesta investigação utilizamos os métodos da sedimentação espontânea e o de Kato, pois foram implantados há alguns anos no Instituto Adolfo Lutz. O método da sedimentação 
espontânea tem sido um dos mais utilizados em inquéritos epidemiológicos, devido à sua eficiência na detecção de um maior número de formas parasitárias, sendo também de execução simples e de baixo custo. O método de Kato, além de ser sensível para detectar ovos de helmintos, possibilita um controle de qualidade dos exames parasitológicos já realizados, pois as lâminas podem ser guardadas para uma análise posterior.

Dentre os enteroparasitas encontrados neste estudo, merece destaque Giardia lamblia e Entamoeba histolytical Entamoeba dispar, pelo potencial para a produção de um grande número de formas infectantes - os cistos, que estes agentes possuem $^{2}$. Os demais protozoários observados (Entamoeba coli, Endolimax nana e Iodamoeba butschilii) são comensais, não sendo patogênicos. Schistosoma mansoni, agente etiológico da esquistossomose mansônica, não é transmitido pela via fecal-oral. Os cistos de G. lamblia e E. histolytica/E. dispar são eliminados juntamente com as fezes para o meio-ambiente, sendo que a transmissão humana ocorre pela via fecal-oral, ou seja, através da ingestão de água e alimentos contaminados. Já foram relatados surtos de gastroenterite associados à contaminação de alimentos por estes protozoários ${ }^{16,17}$. Menos de $20 \%$ dos casos de infecções intestinais pela Giardia nos adultos, apresentam sintomatologia. Os portadores assintomáticos podem excretar os cistos nas fezes durante semanas ou meses, os quais são bastante resistentes, permanecendo viáveis por longo período de tempo no meio-ambiente, contaminando a água e os alimen-

Tabela 1 - Enteroparasitas e leveduras entre 23 manipuladores do município de Ribeirão Preto, SP, Brasil.

Table 1 - Enteroparasites and yeasts in 23 food handlers in the city of Ribeirão Preto, SP, Brazil.

\begin{tabular}{|c|c|c|c|c|c|}
\hline Manipulador $\mathrm{N}^{\circ}$ & Sexo & Idade & Local de Trabalho & Enteroparasitas & leveduras \\
\hline 1 & M & 18 & restaurante & - & Candida parapsilosis \\
\hline 2 & $\mathrm{~F}$ & 51 & padaria & - & Candida tropicalis \\
\hline 3 & $\mathrm{~F}$ & 37 & restaurante & - & - \\
\hline 4 & $\mathrm{~F}$ & 30 & lanchonete & - & Candida parapsilosis \\
\hline 5 & $\mathrm{~F}$ & 45 & hotel & - & - \\
\hline 6 & M & 36 & mercado & - & - \\
\hline 7 & $\mathrm{~F}$ & 30 & escola & - & Candida $\mathrm{sp}^{\mathrm{a}}$ \\
\hline 8 & $\mathrm{~F}$ & 20 & lanchonete & - & - \\
\hline 9 & $\mathrm{~F}$ & 54 & restaurante & - & Candida parapsilosisa \\
\hline 10 & $\mathrm{~F}$ & 60 & bar & E. nana & - \\
\hline 11 & $\mathrm{~F}$ & 56 & hospital & - & - \\
\hline 12 & $\mathrm{~F}$ & 36 & lanchonete & - & Candida parapsilosis \\
\hline 13 & M & 52 & restaurante & - & Candida parapsilosis \\
\hline 14 & $\mathrm{~F}$ & 34 & bar & - & - \\
\hline 15 & $\mathrm{~F}$ & 29 & lanchonete & - & - \\
\hline 16 & $\mathrm{~F}$ & 47 & restaurante & E.nana & Candida sp \\
\hline \multirow[t]{2}{*}{17} & M & 20 & restaurante & E. coli, E. histolytica/E. dispar, & \\
\hline & & & & $\begin{array}{l}\text { G. Iamblia, E. nana, } \\
\text { I. butschilii }\end{array}$ & Candida tropicalis \\
\hline 18 & $\mathrm{~F}$ & 34 & panificadora & S. mansoni & Candida $\mathrm{sp}^{\mathrm{a}}$ \\
\hline 19 & $\mathrm{~F}$ & 44 & lanchonete & - & - \\
\hline 20 & M & 27 & restaurante & - & Candida sp \\
\hline 21 & $\mathrm{~F}$ & 33 & restaurante & - & - \\
\hline 22 & M & 58 & padaria & - & Candida tropicalis \\
\hline 23 & $\mathrm{~F}$ & 53 & supermercado & - & - \\
\hline
\end{tabular}

${ }^{a}$ micológico com exame direto negativo e cultura positiva 
tos $^{2}$. Os cistos de ambos os parasitas são resistentes às concentrações de cloro usadas habitualmente no tratamento da água para o abastecimento público, o que favorece a veiculação hídrica destes parasitas ${ }^{2}$. Surtos em vários países, causados pela ingestão de água de abastecimento contaminada, têm sido registrados ${ }^{2}$. A contaminação hídrica pela Giardia também é preocupante, devido a irrigação de produtos alimentares, como verduras, legumes e frutas ${ }^{18}$. O monitoramento da contaminação de alimentos por parasitas é difícil, pois geralmente eles estão presentes no alimento em pequeno número, dificultando a visualização microscópica dos mesmos, e não há meios de enriquecimento apropriados para a recuperação de parasitas. A aplicação de métodos mais eficazes de detecção, como os ensaios imunológicos com anticorpos monoclonais e métodos moleculares como o PCR são inacessíveis a muitos laboratórios devido a seu alto custo $^{2}$. Como a demonstração microscópica é difícil, a confirmação dos surtos alimentares causados por parasitas, geralmente é feita mais por critérios epidemiológicos do que por laboratoriais. Por este motivo, acredita-se que exista uma sub-notificação das doenças transmitidas por alimentos contaminados por parasitas e, conseqüentemente, uma carência de dados estatísticos, dificultando o dimensionamento do problema e o planejamento de ações por parte dos órgãos de vigilância para monitorar a ocorrência de doenças parasitárias associadas aos alimentos.

Entre as micoses cutâneas, a onicomicose é uma manifestação muito comum na prática dermatológica, sendo relativamente freqüente entre as mulheres, particularmente as que estão em maior contato com a água, como cozinheiras e lavadeiras, e entre os homens é mais comum nos lavadores de louças, manipuladores de frutas, jardineiros e outros. O quadro clínico é bastante variável, e na maioria dos casos observa-se um processo de paroníquia primitivo onde as lesões no limbo são secundárias podendo eliminar material sorológico ou fracamente pu- rulento, revelando ao exame microscópio numerosas leveduras, algumas vezes associadas a Staphylococcus ${ }^{19}$. Segundo o CDC ${ }^{1}$, muitas cepas do $S$. aureus têm uma grande capacidade de contaminar alimentos, devido à produção de uma enterotoxina e à habilidade de crescer em uma ampla faixa de temperatura $\left(7\right.$ a $\left.48^{\circ} \mathrm{C}\right)$ e de $\mathrm{pH}(4,2$ a 9,3). Além disso, entre 30 a $50 \%$ da população humana é portadora da cepa capaz de produzir toxina enterotoxigênica. Em nosso meio já foram relatados estudos vinculando os manipuladores na contaminação de alimentos por esta bactéria ${ }^{20}$. Portanto, a importância do diagnóstico das onicomicoses neste grupo profissional não se limita somente à questão da higiene do trabalhador, assumindo relevância quanto à possibilidade de infecções secundárias por bactérias produtoras de intoxicações alimentares ${ }^{12,20}$.

A casuística do estudo demonstrou a presença de leveduras do gênero Candida sp como sendo a mais freqüente $(34,8 \%)$. Araújo et al. ${ }^{11}$, num extenso trabalho sobre onicomicoses por fungos emergentes, observou maior freqüência de candidíases (49\%) corroborando o presente trabalho.

Entre as 12 amostras com cultura positiva, foram evidenciadas leveduras no exame direto em 08 amostras, sugerindo tratar-se de apenas uma colonização e não uma infecção nas quatro amostras com apenas culturas positivas.

Embora C. albicans seja a levedura mais envolvida nas infecções fúngicas, a C. parapsilosis foi a espécie mais isolada. Midgley \& Moore (1998) ${ }^{21}$ também encontraram C. parapsilosis com maior freqüência entre as candidíases. Porém, deve-se levar em conta que a população estudada nos diferentes trabalhos não tinha a mesma característica.

O papel do Laboratório de Saúde Pública é identificar os agentes etiológicos, dando suporte para que a Vigilância Sanitária possa executar ações preventivas e corretivas. No entanto, a existência de legislação sanitária exigindo do manipulador de alimentos a obtenção e renovação de carteira de saúde, não é suficiente para garantir a 
inocuidade dos alimentos. É necessário que haja uma fiscalização eficiente da carteira de saúde por parte dos órgãos de vigilância, principalmente dos trabalhadores autônomos. No município de Ribeirão Preto é visível nos últimos anos o aumento do comércio ambulante de alimentos, representado pelos carrinhos de lanche, suco, sorvete e outros. Portanto, na prevenção da contaminação de alimentos é indispensável a realização de Programas de Treinamento que proporcionem a educação e formação em higiene dos alimentos para os manipuladores, bem como para os proprietários de estabelecimentos que fabricam e comercializam alimentos. A conscientização do manipulador poderia levá-lo a exercer seu trabalho com mais responsabilidade e ética, beneficiando os consumidores que teriam uma oferta de alimentos mais seguros e de melhor qualidade. Por outro lado, o manipulador, ciente da importância de obter e renovar sua carteira de saúde, teria acesso a assistência médica e exames periódicos, melhorando suas condições de saúde. Vários autores já enfatizaram a importância da melhoria da qualificação do manipulador através da educação em saúde como sendo um fator relevante na redução e prevenção das doenças alimentares ${ }^{22,23}$. A utilização do sistema HACCP ("Hazard Analysis and Critical Control Point”) e a fiscalização de sua aplicação nos estabelecimentos de alimentos também se faz necessária para garantir a inocuidade dos alimentos.

\section{Conclusões}

Para evitar as doenças transmitidas por alimentos é necessária a implementação conjunta de várias ações como a fiscalização da carteira de saúde, a promoção de educação em higiene de alimentos para os manipuladores e proprietários, e a implantação do sistema HACCP nos estabelecimentos de alimentos. Neste contexto, é de suma importância a identificação dos portadores de agentes patogênicos que possam ser propagados para os alimentos através da manipulação.

Devido ao fato de a inocuidade dos alimentos ser relevante para a saúde pública, os autores sugerem a realização de outros estudos que possam colaborar na prevenção da ocorrência de doenças de transmissão alimentar,

\section{Referências}

1. Centers for Disease Control and Prevention. Diagnosis and management of foodborne illnesses: a primer for physicians. MMW Recomm Rep 2004; 53: 1-33.

2. Orlandi PA, Chu D-MT, Bier JW, Jackson JG. Parasites and the food supply. Foodtechnology 2002; 56: 72-81.

3. Käferstein FK. Food safety: the fourth pillar in the strategy to prevent infant diarrhoea. Bull World Health Organ 2003; 81: 842-3.

4. [OPS] Organização Panamericana de Saúde. Sistema Regional de Información para la Vigilancia de las Enfermedades Transmitidas por Alimentos. Disponível em: URL<http://www.panalimentos.org/sirveta/e/ index.htm $>$ [acessado em 29 de agosto de 2005]

5. SÃO PAULO. Secretaria de Estado da Saúde. Centro de Vigilância Epidemiológica. Divisão de Doenças de Transmissão Hídrica e Alimentar. Disponível em URL<http//www.cve.saúde.sp.gov.br/htm/dta menu.htm $>$ [acessado em 9 de agosto de 2005]
6. Adams VJ, Markus MB, Adams JF, Jordan E, Cutis B, Dhansay MA et al. Paradoxical helminthiasis and giardiasis in Cape Town, South Africa: epidemiology and control. Afr Health Sci 2005; 5:131-6.

7. Celiksoz A, Guler N, Guler G, Oztop AY, Degerli S. Prevalence of intestinal parasites in three socioeconomically-different regions of Sivas, Turkey. $J$ Health Popul Nutr. 2005; 23: 184-91.

8. Verle P, Kongs A, De NV, Thieu NQ, Depraetere K, Kim HT et al. Prevalence of intestinal parasitic infections in northern Vietnam. Trop Med Int Health 2003; 10:961-4.

9. Morales-Espinoza EM, Sanchez-Perez HJ, Garcia-Gil M del M, Vargas-Morales G, Mendez-Sanchez JD, PerezRamirez M. Intestinal parasites in children, in highly deprived areas in the border region of Chiapas, Mexico. Salud Publ Mex 2003; 45:379-88.

10. Saldiva SR, Silveira AS, Philippi ST, Torres DM, Mangini AC, Dias RM et al. Ascaris-Trichuris association and malnutrition in Brazilian children. Perinat Epidemiol 1999, 13:89-98. 
11. Araújo AJG, Bastos OMP, Souza MAJ, Oliveira JC. Onicomicoses por fungos emergentes: análise clínica, diagnóstico laboratorial e revisão . An Bras Dermatol 2003; 78: 445-55.

12. Sabioni JG, Hirooka EY, Souza MLR. Intoxicação alimentar por queijo minas contaminado com Staphylococcus aureus Rev Saúde Pública. 1998; 22: 45861 .

13. SÃO PAULO. Secretaria de Estado da Saúde. Portaria CVS-6/99 de 10 de março de 1999. Aprova o regulamento técnico que estabelece os parâmetros e critérios para o controle higiênico-sanitário em estabelecimentos de alimentos. Disponível em: URL< http:/ / www.saude.sp.gov.br/html/fr links.htm > [ acessado em 29 de agosto de 2005]

14. Kato K, Miura M. Comparative examinations. Jap J parasit $1954 ; 3: 35$.

15. Kurtzman PC, Fell JW. The yeasts: A Taxonomic Study. Amsterdam; Elservier; 1998.

16. De Lalla F, Rinaldi E, Santoro D, Nicolin R, Tramarin A. Outbreak of Entamoeba histolytica and Giardia lamblia infections in travelers returning from the tropics.

Infection 1992; 20: 78-82.
17. Mintz ED, Hudson-Wragg M, Mshar P, Cartter ML, Hadler, JL. Foodborne giardiasis in a corporate office setting. J Infect Dis 1993; 167: 250-3.

18. Ashbolt NJ. Microbial contamination of drinking water and disease outcomes in developing countries. Toxicology 2004; 198: 229-38.

19. Lacaz CS, Porto E, Martins JEC. Micologia Médica. In: Fungos, actinomicetos e algas de interesse médico. Editora Savier; 1984. p. 163-165.

20. Ribeiro, EGA, Martins AMB, Oliveira MA, Silva P, Errera MC, Carloni MC et al. Identificação do agente causal de um surto de toxinfecção alimentar. Hig Alim 1999; 13: 8890 .

21. Midgley G, Moore MK. Onychomycosis. Rev Iberoam Micol 1998; 15:113-7

22. Germano MIS, Germano PML, Kamei CAK, Abreu ES, Ribeiro ER, Silva KC et al. Manipuladores de alimentos: Capacitar? É preciso. Regulamentar?... Será preciso???. Hig Alim 2000; 14:18-22.

23. Ungar, ML, Germano, MIS, Germano, PML. Riscos e conseqüências da manipulação de alimentos para a saúde pública. Hig Alim 1992; 6:14-7.

Recebido em: 12/05/05

Versão reformulada reapresentada em: 03/10/05

Aprovado em: 10/10/05 